\title{
Making the most of by-catch data: Assessing the feasibility of utilising non- target camera trap data for occupancy modelling of a large felid
}

\author{
Edwards, Sarah ${ }^{1,2} *$, Cooper, Sue ${ }^{3}$, Uiseb, Kenneth ${ }^{4}$, Hayward, Matt ${ }^{5}$, Wachter, Bettina ${ }^{1}$, \\ Melzheimer, Joerg ${ }^{1}$
}

\footnotetext{
${ }^{1}$ Evolutionary Ecology, Leibniz Institute for Zoo and Wildlife Research, Berlin.

${ }^{2}$ Centre for Wildlife Management, University of Pretoria, Pretoria, South Africa.

${ }^{3}$ Greater Fish River Canyon Landscape, Namibia

${ }^{4}$ Scientific Services, Ministry of Environment and Tourism, Windhoek, Namibia.

5 School of Environmental and Life Sciences, The University of Newcastle, Australia.

* corresponding author: sarah@cheetahnamibia.com
}

\begin{abstract}
Camera traps are an increasingly used tool in ecology, having the ability to capture large numbers of photographic records in short survey periods. For many surveys the number of non-target records outweighs those of focal species, making them a potentially rich and often under-utilised data source. Occupancy analysis of non-target data represents a potential way to optimise survey output, whilst increasing 'return on investment'. This study assessed the feasibility of using non-target data from a Hartmann's mountain zebra Equus zebra hartmannae survey in Gondwana Canyon Park, southern Namibia, for occupancy analysis on leopard Panthera pardus. Using a survey design with 15 camera traps at water sources, 26 leopard events were detected over 72 days. Model fit was adequate and produced a modelaveraged occupancy of 0.64 (S.E. 0.36), and a detection probability of 0.24 (S.E. 0.07). Whilst there was a lack of precision in the final occupancy estimate, the study provided valuable pilot data for future surveys. The results highlight the ability of camera traps to obtain information-rich datasets, which, when properly archived, can be used for providing information on a number of ecological topics, ranging far beyond that which the traps were originally deployed for.
\end{abstract}

Keywords: by-catch, camera trap, distribution, leopard, occupancy, Namibia 


\section{Introduction}

Camera traps are an increasingly used tool in the field of ecology and wildlife management (O'Connell, Nichols \& Karanth, 2011). With the potential to capture more than a million photo records during relatively short survey periods (Krishnappa \& Turner, 2014), the methodology is creating a vast amount of new data (Burton et al., 2015). Although surveys often focus on a single species, camera traps inevitably record multiple species, and for many surveys the number of records of non-target species outweighs that of focal species (Kelly \& Holub, 2008). Therefore, for those studies focusing on a single species or research question, for example those estimating abundance, records of non-target species can represent an often under-utilised, yet potentially rich data source (e.g. Pamplin, 2013). Utilisation of non-target species data from camera trap surveys has the potential to optimise scientific output, as well as increasing the 'return on investment' of such surveys, which are often costly to run (Pamplin, 2013). In order to make use of non-target data, researchers may apply the same analytical techniques to all species recorded on camera traps for which sufficient sample sizes were achieved. In a review of 266 camera trap studies published between 2008 and 2013, Burton et al. (2015) found $26.6 \%$ of 42 density surveys to use the same survey design to estimate density for multiple species. For example, Harihar et al., (2010) used a camera trap data set originally deployed for tiger Panthera tigris to estimate the density of striped hyena Hyaena hyaena.

Occupancy modelling represents another practical solution for utilising non-target species data, given the survey design meets model assumptions. Occupancy is defined as the proportion of sites (i.e. camera trap sites) occupied by a species, estimated whilst accounting for imperfect detection (Mackenzie et al., 2006). It is an important and extensively used concept in ecology (Mackenzie and Royle, 2005), and is routinely used for modelling of habitat relationships and metapopulation studies (Guillera-Arroita, Ridout \& Morgan, 2010). In addition to estimating occupancy, models can be used to explore the influences of environmental variables on species distributions and aid in understanding patterns of habitat use (Srivathsa et al., 2014). Occupancy analysis requires simple, spatially or temporally repeated presence/absence data, without the need for individual identification, making it compatible for use with species without natural pelage patterns (Mackenzie et al., 2006), and when identification of individuals may not be possible. 
Occupancy modelling has been used extensively across a variety of species and habitats, its popularity owing partially to the fact that surveys typically require a lower number of camera trap sites than abundance surveys and are therefore considered less expensive to run (Mackenzie et al., 2006). Occupancy models also explicitly account for imperfect detection, and are robust to temporal or spatial variation in detection probability (Mackenzie et al., 2006). Furthermore, occupancy models estimate detection probability; the probability of detecting a species at least once during a sampling occasion, provided the site is occupied (Mackenzie et al., 2006). Estimates of detection probability can be used to calculate the sampling effort needed to produce precise occupancy estimates when designing future studies (Mackenzie \& Royle, 2005).

Originally, occupancy models were designed for sedentary animals, occurring in discrete patches which would be closed to changes in occupancy during the sampling period (Mackenzie et al., 2002). In such a scenario, any sampling occasion in which the species was not detected was due to error, and detection probability could be interpreted as reflecting error only (Efford \& Dawson, 2012). However, today, occupancy surveys are used for a wide variety of species, including highly mobile species occurring in continuous habitats, which typically range beyond the detection zone of the camera trap (Burton et al., 2015). When such species are not detected during a sampling occasion, it may be due to error, or the animal moving away from the camera trap, and not being available for capture at that time. Therefore, for occupancy surveys of highly mobile species, the detection probability conflates both error and detection probability. Given that the occupancy framework explicitly models the detection process separately from that of occupancy, occupancy models for mobile species must be used with caution (Efford \& Dawson, 2012), with a full understanding of the true meaning of detection probability estimations and variance.

Like all models, single season occupancy models have a set of assumptions which if violated may lead to biased estimators and incorrect inferences regarding model results. Occupancy models have four main critical assumptions: (1) there are no changes in the occupancy status of each camera trap site during the survey period, unless these changes are considered nonMarkovian (Mackenzie et al. 2006), however open population and dynamic occupancy models have been developed, e.g. Dail and Madsen (2011). It is recommended that survey length covers two to three months to not violate the assumption for large felids (Karanth \& Nichols, 1998). However, this to some degree depends on the detection probability of the species because higher detection probabilities require fewer survey days (Rovero et al., 
2013). Mackenzie et al. (2002) recommend a detection probability of 0.15 or higher, as the threshold for producing accurate occupancy estimates. (2) there is an equal probability of occupancy across sites, i.e. each site has an equal probability of being occupied by the target species, and (3) there is an equal detection probability across sites. If assumptions (2) and (3) are not met, then any heterogeneity must be modelled using environmental covariates (Mackenzie et al. 2006). This can be achieved by placing camera traps in a variety of habitats, and by carefully choosing environmental covariates for modelling probability of occupancy based on the ecology of the species. (4) the detection of species and detection histories are independent at each site (Mackenzie et al. 2006), or can be modelled as spatial random effects when using spatially explicit occupancy models (Johnson et al., 2013). The assumption of spatial independence may be achieved by placing camera traps at a distance larger than the diameter of the average home range of the target species, thus avoiding spatial auto-correlation (Rovero et al., 2013). For species in which individual identification is possible, the use of individual identity may be useful in checking assumptions are met. For example, the assumption of spatial independence, could be checked by ascertaining if individuals were recorded at more than one site. Additionally, the closure assumption of Stanley and Burnham (1999) could be used to check that demographic closure occurred within the study period. When demographic closure is not achieved, changes in the occupancy status of sites may occur due to individuals immigrating into and emigrating out of the study area, and thus violate the assumption.

Surveys placing camera traps at water sources have the potential to record a wide variety of species, particularly in arid environments, where surface water is scarce (Edwards, Gange \& Wiesel, 2016). They therefore represent particularly promising datasets for which to investigate the potential of utilising non-target data, and given the diversity of species visiting water sources, a chance to run occupancy models on species very different from those the camera traps were originally deployed for. The Mountain Zebra Project, which focuses on Hartmann's mountain zebra Equus zebra hartmannae in the Gondwana Canyon Park (GCP), southern Namibia, positioned camera traps exclusively at artificial, permanent water sources to obtain images suitable for individual zebra recognition (Gosling, 2015), taking advantage of the water dependency of the species (Novellie, 2008). These water sources were installed by the park management as a way of provisioning water for wildlife, and are regularly used by zebra, and a number of other species. Leopard Panthera pardus was amongst the nontarget species recorded. Given the challenges associated with surveying large carnivores, 
such as low densities and large home ranges (Long et al., 2007), leopard represents an interesting candidate for occupancy analysis.

Leopards have the widest distribution of any large felid in the world (Stein \& Hayssen, 2013), with their well documented behavioural plasticity allowing them to persist in a wide variety of habitats, as well as near to major cities (Athreya et al., 2013). Despite this, a recent review found leopards to occupy just 25 - $37 \%$ of their historical range, with only $17 \%$ of the extant range having some degree of conservation protection (Jacobson et al., 2016). A lack of empirical field data on distribution for the species has been noted (Jacobson et al., 2016), motivating the need for further studies, especially in previously unsurveyed areas. Within Namibia, leopards are utilised by the trophy hunting sector, and are a species frequently involved in human-wildlife conflict (Lindsey et al., 2013). As such their population must be carefully monitored to ensure long-term survival (Baker, 2004; Thirgood \& Rabinowitz, 2005). The government of Namibia and its Ministry of Environment and Tourism (MET) actively promote and manage conservation, and have a long tradition of initiating carnivore surveys. In 2012, five areas across the country were surveyed for leopard density (Stein et al., 2011).

This study aimed to investigate the feasibility of using the non-target leopard dataset from the Hartmann's mountain zebra survey of the GCP for performing occupancy analysis. Given the significant differences in the ecology of the two species, the utilisation of leopard bycatch data for occupancy modelling may be particularly challenging, especially when ensuring model assumptions are met. Due to the survey being conducted in a managed, conservation and tourism area, the influences of environmental variables, including those related to park management, were assessed for their influence on occupancy and detection probability. The primary objectives of this study were to (i) determine occupancy and detection probability of leopard, whilst ensuring fit of the global model, i.e. a model containing all potential covariates, is adequate and model assumptions were met, (ii) examine the influences of environmental variables, including those related to park management, on leopard occupancy and (iii) to use the final occupancy and detection probability estimates to estimate the total survey effort needed to achieve an occupancy estimate with a SE of 0.05 in the same or similar habitat, following Mackenzie and Royle (2005). 


\section{Methods}

\subsection{Study site}

The Gondwana Canyon Park, established in 1995, covers a total area of $1,300 \mathrm{~km}^{2}$ and is situated east of the settlement of Grünau in southern Namibia. The park is managed to conserve the ecosystems and biodiversity within it, and when necessary rehabilitate areas of land degradation. The southern section of the park is Nama Karoo Biome, and straddles the transition area into Succulent Karoo Biome. With an annual median rainfall of $100.1 \mathrm{~mm}$, the park is classified as desert (National Drought Task Force, 1997), with the majority of rain falling between January and April, and ecologically significant winter (July to September) rainfall occurring on average every four years. The park shares an approximately $70 \mathrm{~km}$ long un-fenced western border with the Ai-Ais/Hunsberg National Park $\left(310 \mathrm{~km}^{2}\right)$, with the remaining border shared with commercial farmlands. Eighteen artificial water sources are maintained in the GCP, and wildlife species include, apart from Hartmann's mountain zebra and leopard, spotted hyena Crocuta crocuta, caracal Caracal caracal, cheetah Acinonyx jubatus, oryx Oryx gazella and springbok Antidorcas marsupialis. The GCP contains three tourist lodges, with the main access road entering the park in the northeast and western side of the park.

\subsection{Survey design}

Camera traps were set across the GCP at artificial water sources (Figure 1) to record Hartmann's mountain zebra (Ministry of Environment and Tourism research permit \#2100/2015), from January 2015 to July 2016. A subset of data were used for occupancy analysis so as not to violate the assumption of species closure. Data from 15 camera traps were collected in the rainy season during 72 days from 18th January to 29th March 2016, representing a period when most camera traps were active throughout the study period. Camera traps had a mean distance of $9.62 \mathrm{~km}( \pm$ SE 1.41) between them, which is larger than the mean $95 \%$ minimum convex polygon home range diameter $(9.52 \mathrm{~km})$ of leopard in north central Namibia (Stein et al., 2011), classing them as spatially independent. However, leopard home ranges are known to vary widely in size across habitats (Stein and Hayssen, 2013), and no leopard home range size estimates are available for the study area, giving rise to the potential of sites not being spatially independent. No instances of an individual visiting more than one water source were found in the entire dataset, however, given the number of blurry images and instances where only one flank of an individual was recorded, the 
possibility cannot be ruled out. In cases where the spacing of camera traps is smaller than the average home range of a species, occupancy can be used as a measure of habitat use, rather than 'true occupancy' (Mackenzie, 2005), which must therefore be considered in this study.

Figure 1: Locations of camera traps, artificial and permanent water sources, lodges and houses, and access roads to the lodges in Gondwana Canyon Park, southern Namibia.

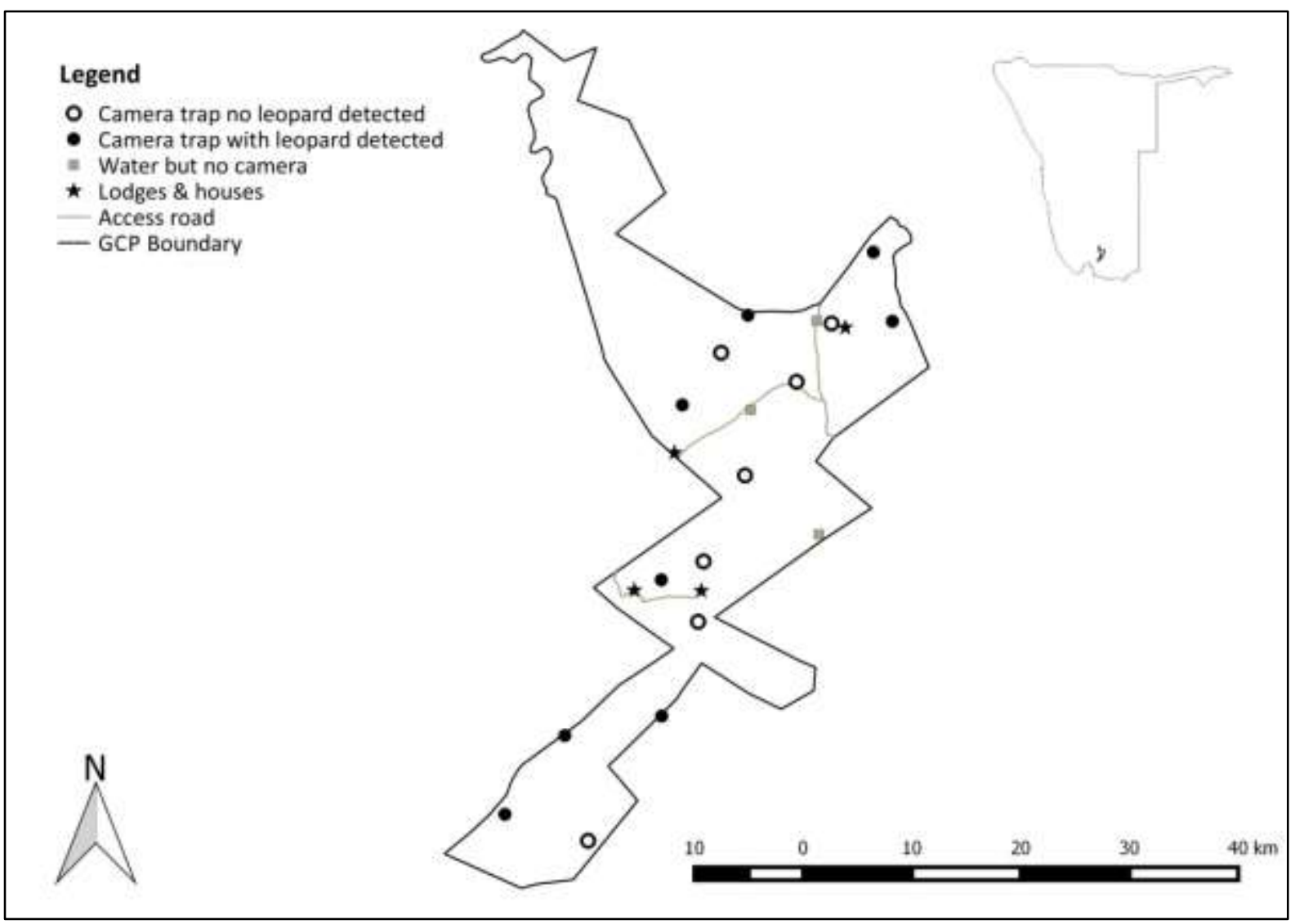

A variety of camera trap makes and models were used; LTI Acorn LTI-5310 (LTI Acorn, Shenzen City, China), Moultrie M-880 (Ebsco Industries Inc, AL, USA), Spartan SR1-IR (HCO Outdoors, GA, USA), Scoutguard SG550 (HCO Outdoors, GA, USA), Covert Scouting camera 2014 MP8 (Covert Scouting Cameras, KY, USA) and Ranger Trail camera BN073 (Ranger Outdoors). Such a range of camera trap makes and models has the potential to bias data, due to the different detectabilities of camera trap types (Kelly \& Holub, 2008). However, given that leopard visiting water holes are assumed to do so to drink (and all photos from this study showed leopards either drinking or directly approaching the water's edge), the time spent in front of the cameras, combined with the large body size, should be sufficient for even the slower trigger speeds to capture an image, and therefore was not considered an issue. All camera traps were set to the highest image quality each model allowed, and a medium sensitivity to avoid recording smaller animals such as birds. Each 
camera trap was located approximately 5 - $7 \mathrm{~m}$ from the water source, on a pole, or a tree when available. No camera traps were set in the north-western section of the park, due to this area being devoid of artificial water sources.

\subsection{Covariates}

Six potential covariates were selected for this study (Table 1). They were related to leopard ecology and also to anthropogenic factors, thereby allowing the influences of management practices on site occupancy to be assessed. GPS positions of camera traps, artificial, permanent water sources, tourist lodges and staff houses (the latter two collectively referred to as human habitation) within the park were plotted in QGIS 2.8.4-Wien (Quantum GIS Development Team, 2015), along with the park boundary and access roads to the lodges. Euclidean distances were calculated between camera traps and nearest neighbour artificial water source, human habitation, access roads and the park boundary. Elevation was extracted from Google Earth (Google, 2016). Only access roads leading to the three lodges of the GCP were considered, as smaller, infrequently used trails, i.e. those leading to staff houses, are not known to affect the behaviour of carnivores (Mladenoff \& Sickley, 1998). Habitat type at each camera trap site was taken from maps produced within the park's management plan, which included five main habitat types: (i) incised rocky hills, (ii) sandy or gravel plains, (iii) plain with koppies (small hills in a generally flat area), (iv) plateau and (v) river (Anon, 2015). Habitat type entered as a dummy variable into PRESENCE 7.0 (Hines, 2006), and all continuous covariates were standardised to $\mathrm{Z}$ scores prior to analysis, and summary statistics can be found in Table 2 .

Table 1: Environmental covariates included in potential candidate occupancy models

\begin{tabular}{ll}
\hline Covariate & Definition \\
\hline Water & Straight line distance from camera trap site to the nearest neighbouring water source \\
Road & Straight line distance from camera trap site to the nearest park access road \\
Boundary & Straight line distance from camera trap site to the park boundary \\
Human habitation & Straight line distance from camera trap site to the closest house or tourist lodge \\
Elevation & Elevation in metres above sea level of the individual camera trap site \\
Habitat & Five habitat types defined from Gondwana Canyon Park management document \\
\hline
\end{tabular}


Table 2: Summary statistics of continuous covariates

\begin{tabular}{lrr}
\hline Covariate & Mean $( \pm$ S.D) & \multicolumn{1}{l}{ Range } \\
\hline Water $/ \mathrm{km}$ & $7.49(2.75)$ & $1.07-10.96$ \\
Road $/ \mathrm{km}$ & $5.76(4.52)$ & $0.35-16.20$ \\
Boundary/km & $2.90(2.17)$ & $0-7.60$ \\
Human habitation/km & $9.88(8.16)$ & $0.10-27.55$ \\
Elevation/m & $879.47(130.26)$ & $628-1,165$ \\
\hline
\end{tabular}

To avoid multicollinearity, Spearman's rank correlations were used to test for nonindependence between covariate pairs in R 3.1.1 (R Core Development Team, 2014). Covariate pairs with $(|r|>0.80)$ would considered to be highly correlated, and in such cases one covariate from the pair would be excluded from further analysis (Long et al., 2011). However, Spearman's correlation tests showed no evidence of multicollinearity, therefore no covariates were dropped from the first stage of analysis. Leopards were detected 26 times, allowing 2.6 (rounded to three) covariates to be included within the candidate models, following the $\mathrm{n} / 10$ rule of Anderson (2008) to avoid model over-fitting. To reduce the six potential covariates to three, six occupancy models were run, each including a different individual covariate for influencing occupancy, and the three top ranking models, based on AIC values, were used to select the covariates to include within the candidate model set.

\subsection{Occupancy analysis}

A high proportion of blurry images (0.42) made individual identification impossible, and a criteria of a minimum of 30 minutes between consecutive leopard photos was employed for defining independent events, following O'Brien, Kinnaird \& Wibisono (2003). However, the minimum time between consecutive leopard visits to a site was eight hours. A standard "Xmatrix format" detection history (Otis et al., 1978) was then constructed, using 12, six day sampling occasions to avoid having too many 'non-detections' within the sampling occasions, which is known to result in a reduced accuracy of model estimates (Mackenzie \& Royle, 2005). Given that leopard are primarily nocturnal, a sampling day was defined as starting at 12:00 midday, and ending 11:59 the following day. This should ensure avoidance of the 'midnight problem', whereby an animal visiting a camera trap either side of midnight in a single night would be recorded present during two consecutive nights (Jordan, Barrett \& Purcell, 2011). Sampling occasions with missing data, i.e. cameras were not active due to dead batteries etc, were coded as ".", and accounted for 43 (0.23) of the 180 sampling occasions. The detection history matrix, along with a covariate matrix were imported into 
PRESENCE 7.0 (Hines, 2006), in order to produce occupancy and detection probability estimates, as well as to explore the effects of environmental covariates on model parameters, following Mackenzie et al. (2006). Single-species/single-season occupancy models were used, which assume demographic closure (Mackenzie et al., 2006). Hence a 72 day survey duration was used, as this survey length falls within the recommended closed period for large felids (Karanth \& Nichols, 1998).

A two-step process was used to construct the final candidate model set (Mackenzie et al., 2006). Firstly, the effect of covariates, both individually and in combination, on detection probability were assessed, whilst keeping occupancy constant. AIC values of covariate models were compared to those from the simplest model for which detection probability was kept constant, to assess which gave a better fit. In the second step, the top-ranking model for detection probability was selected from the set of candidate models, and was combined with the candidate models for occupancy. Occupancy was allowed to vary with covariates, either individually or in combination, and compared to the simplest model for which occupancy was kept constant. Goodness of fit of the global model, i.e. a model containing all potential covariates, was tested using 10,000 parametric bootstraps within PRESENCE 7.0, as well as an estimate of $\hat{c}$, the variance inflation factor. The value of $\hat{c}$ gives a measure of variation in the data, indicating whether significant over or under dispersion is present. The $\hat{c}$ value should be as close to 1 as possible, with results over or under 1 indicating a poor fit for the data (Cooch \& White, 2006).

Ranking of candidate models was based on the Akaike information criterion (AIC) values (Burnham \& Anderson, 2002) produced by PRESENCE 7.0. AIC values were not corrected for small sample size, given the lack of agreement on the definition of the effective sample size in occupancy models, which could mean the number of camera trap sites or the number of detections, therefore AIC values were used following Mackenzie et al. (2006). The most parsimonious models were defined as those with $\triangle \mathrm{AIC}$ values $<2.0$, and their covariates interpreted as having the strongest influence on model parameters (Burnham \& Anderson, 2002). As no single model contained $>90 \%$ of the support within the candidate set, i.e. individual AIC weights $(w i)<0.90$, weighted model-averaging was used to produce the final leopard occupancy and detection probability estimates, using the models with AIC weights larger than 0.01 (Linkie et al., 2007). AIC weights were used to assess the relative importance of variables within each model, showing the weight of evidence in favour of the model given the model set (Burnham \& Anderson, 2002). As no single model contained the 
majority of support, a $90 \%$ confidence model set was identified, i.e. those models with wi values summing to $\geq 0.90$. The relative importance of each covariate was then assessed by summing the normalised wi values for each model in which that particular covariate occurred (Mackenzie et al., 2006).

\subsection{Design of future occupancy surveys}

To estimate the optimum number of sampling occasions needed for future surveys, the final occupancy and detection probabilities were applied to the Table 1 of Mackenzie and Royle (2005), assuming the financial and time cost of running each sampling occasion is equal to the initial sampling occasion. The optimum number of camera trap sites needed to achieve an occupancy estimate with a precision of $\mathrm{SE}=0.05$ was also calculated, following equation 3 in Mackenzie and Royle (2005), using a standard survey design.

\section{Results}

\subsection{Photo statistics}

A total of 27 mammal and 14 bird species were detected by the camera traps. Twenty-six independent leopard events were detected during the 137 active trap sampling occasions, giving a mean encounter frequency of 0.19 using a six day sampling occasion. Camera traps failed for a 43 sampling occasions due to problems with baboon Papio cynocephalus interference and battery issues. Leopard events occurred across the park, with eight of the 15 camera traps detecting leopard (as shown in Figure 1), giving a naive occupancy estimate of 0.53 .

\subsection{Occupancy analysis}

Ranking AIC values showed distance to nearest neighbouring water source, distance to access road and elevation to be the three most influential covariates out of the original set of six. Goodness of fit testing of the global model provided no evidence for poor fit to the data (probability of test statistic $\geq$ observed $=0.27$ ). Additionally, no evidence for under or over dispersal was seen, with $\hat{c}$ estimated at 1.08. Such results suggest heterogeneity in occupancy and detection probabilities could be modelled using environmental covariates, meaning critical assumptions (2) and (3) were met.

Using models for which occupancy was kept constant (i.e. $\psi()$.$) , candidate models showed$ distance to nearest neighbouring water source to be the covariate with the strongest influence 
on detection probability (Table 3$)$. Inspection of AIC values showed the model $\psi(\operatorname{Road})$, $p$ (Water), was the top ranking model (Table 4$)$, giving an occupancy estimate of $0.63( \pm \mathrm{SE}$ $0.69)$ and a detection probability of $0.24( \pm$ SE 0.07$)$.

Table 3: Leopard occupancy detection models

\begin{tabular}{lrrrrrr}
\hline Model $^{\mathbf{a}}$ & \multicolumn{1}{c}{ AIC } & \multicolumn{1}{c}{$\boldsymbol{\Delta A} \mathbf{A C}$} & $\boldsymbol{w i}$ & $\mathbf{K}^{\mathbf{b}}$ & $\boldsymbol{- 2}^{*} \mathbf{L o g L i k e}$ & $\boldsymbol{p} \pm \mathbf{1 ~ S E}$ \\
\hline$\psi(),$.$p (Water)$ & 103.04 & 0 & 0.9024 & 2 & 99.04 & $0.24( \pm 0.07)$ \\
$\psi(),. p()$. & 109.43 & 6.39 & 0.037 & 4 & 101.43 & $0.31( \pm 0.06)$ \\
$\psi(),$.$p (Elevation)$ & 111.27 & 8.23 & 0.0147 & 2 & 107.27 & $0.38( \pm 0.08)$ \\
$\psi(),$.$p (Road)$ & 111.77 & 8.73 & 0.0115 & 2 & 107.77 & $0.41( \pm 0.05)$ \\
$\psi(),$.$p (Water \& Elevation)$ & 111.96 & 8.92 & 0.0104 & 3 & 105.96 & $0.32( \pm 0.07)$ \\
$\psi(),$.$p (Water \& Road)$ & 112.01 & 8.97 & 0.0102 & 2 & 108.01 & $0.36( \pm 0.06)$ \\
$\psi(),$.$p (Road \& Elevation)$ & 112.15 & 9.11 & 0.0095 & 3 & 106.15 & $0.35( \pm 0.04)$ \\
$\psi(),$.$p (Water, Road \& Elevation)$ & 113.72 & 10.68 & 0.0043 & 3 & 107.72 & $0.32( \pm 0.06)$ \\
\hline
\end{tabular}

${ }^{a}$ For definitions of model covariates see Table 1

${ }^{b}$ Number of parameters

Table 4: Leopard occupancy $\psi$ and detection probability $p$ from the five top ranking models.

\begin{tabular}{|c|c|c|c|c|c|c|c|}
\hline Model & AIC & $\Delta \mathrm{AIC}$ & Wi & $\mathbf{K}^{\mathbf{b}}$ & $-2 *$ LogLike & $\psi \pm 1 \mathrm{SE}$ & $p \pm 1 \mathrm{SE}$ \\
\hline$\psi($ Road $), p$ (Water $)$ & 93.43 & 0 & 0.4646 & 4 & 85.43 & $0.63( \pm 0.69)$ & $0.24( \pm 0.07)$ \\
\hline$\psi($ Road \& Elevation $), p$ (Water $)$ & 94.83 & 1.4 & 0.2307 & 5 & 84.83 & $0.63( \pm 0.23)$ & $0.24( \pm 0.07)$ \\
\hline$\psi(),$.$p (Water)$ & 95.3 & 1.87 & 0.1824 & 3 & 89.3 & $0.72( \pm 0.06)$ & $0.24( \pm 0.07)$ \\
\hline$\psi($ Elevation $), p$ (Water) & 97.01 & 3.58 & 0.078 & 4 & 89.01 & $0.71( \pm 0.20)$ & $0.24( \pm 0.07)$ \\
\hline$\psi($ Water \& Elevation), $p$ (Water) & 98.52 & 5.09 & 0.037 & 5 & 88.52 & $0.61( \pm 0.28)$ & $0.24( \pm 0.07)$ \\
\hline Weighted model average & & & & & & $0.64( \pm 0.36)$ & $0.24( \pm 0.07)$ \\
\hline
\end{tabular}

${ }^{a}$ For definitions of model covariates see Table 1

${ }^{b}$ Number of parameters

Examination of AIC weights of the candidate model set showed no single model held the majority of support ( $w i>0.90)$, however amongst the five top ranking models $(\Delta \mathrm{AIC}>2.0)$, the first three models contained $87 \%$ of the AIC weight (Table 4). Weighted modelaveraging was used to derive parameter estimates, with leopard occupancy estimated at 0.64 $( \pm$ SE 0.36$)$, and detection probability was $0.24( \pm$ SE 0.07$)$ (Table 4$)$.

Summing wi values showed distance to access roads within the park and site elevation were the covariates with the strongest influence on leopard occupancy (Table 5). Distance to the nearest access road had a 0.69 summed wi $(0.46+0.23$ (Table 4)), with a beta intercept of 2.56 ( \pm SE 1.91), indicating sites further from access roads were more likely to be occupied 
by leopard. Site elevation had a 0.35 summed wi $(0.23+0.08+0.04$ (Table 4$))$, with a beta intercept of -0.82 ( \pm SE 0.39), indicating sites at lower elevations had an increased probability of being occupied by leopard. Distance to nearest neighbouring water source was the only covariate having an influence on detection probability, with a 1.00 summed wi $(0.46+0.23+0.18+0.8+0.4$ (Table 4$))$, with a beta intercept value of $1.10( \pm$ SE 0.39$)$. This indicates camera traps at a given water source had a higher probability of detecting leopards if the nearest neighbouring water source was further away.

Table 5: Influence of covariates on leopard occupancy and detection probability in Gondwana Canyon Park.

\begin{tabular}{lrr}
\hline Covariate & Summed wi & Beta intercept \pm 1 SE \\
\hline Occupancy & & \\
Distance to access road & 0.69 & $2.56( \pm 1.91)$ \\
Site elevation & 0.35 & $-0.82( \pm 0.39)$ \\
\hline Detection probability & & \\
Distance to nearest water & 1.00 & $1.10( \pm 0.39)$ \\
\hline
\end{tabular}

\subsection{Design of future occupancy surveys}

Applying the weighted model-averaged occupancy and detection probabilities to Table 1 of Mackenzie and Royle (2005), 10 sampling occasions were estimated as optimal for future leopard occupancy surveys in habitats similar to the GCP. A total of 112 camera trap sites would be needed to achieve an occupancy estimate with a SE of 0.05 , using this survey design, in the same or similar habitat.

\section{Discussion}

For many camera trap surveys the number of photo records of non-target species outweighs those of target species (Kelly \& Holub, 2008), which are seldom used by the investigator (Sunarto et al., 2013). Although few studies explicitly use non-target data, those that have, have done so to successfully provide essential ecological data such as population estimates (Harihar et al., 2010) or species distributions (Stratford et al., 2016). This study demonstrates the feasibility of utilising camera trap by-catch data, collected during a targeted herbivore survey, for modelling occupancy and probability detection of a large felid; two species with significant differences in their ecology. Despite the relatively low number of independent leopard events recorded, model fit was adequate. However, the model-averaged occupancy estimate had a relatively large standard error. Such results highlight the potential 
of camera trapping surveys to provide estimates regarding the non-target species, as well as providing pilot data necessary to effectively plan future survey designs, thereby increasing the use, and the 'return on investment' of such data (Pamplin, 2013). The study additionally adds to body of literature regarding leopard ecology and distribution, from a previously unsurveyed area, highlighting the appropriateness of camera traps positioned at water sources when surveying leopard in arid environments.

The total of 26 independent leopard events, giving a mean of 3.14 events per 100 camera trap nights, was lower than the 6 events per 100 camera trap nights for leopard on commercial farmlands bordering the Waterberg Plateau Park in north central Namibia (Stein et al., 2011). However, such a result may be expected given the positive relationship between leopard density and rainfall in sub-Saharan Africa (Martin \& de Meulenaer, 1988) and the arid conditions of the park. The study area of GCP was not previously surveyed for leopard density, and the results of landowner questionnaires led to the area being designated as no known occurrence for leopard (Stein et al., 2012). Therefore, the presented study fills such a gap regarding the distribution of leopard in Namibia. The nearest area to the GCP with estimated leopard densities of 0.59 and 0.90 adults $/ 100 \mathrm{~km}^{2}$ come from commercial farmlands approximately $200 \mathrm{~km}$ northwest of the GCP (Edwards et al., 2015). Despite the relatively low number of leopard events, model-averaged detection probability was estimated at $0.24( \pm$ SE 0.07$)$, which is above the recommended threshold of 0.15 required to provide robust occupancy estimates (Mackenzie et al., 2002). Such a relatively high detection probability is likely to result from placing camera traps at artificial water sources which have been shown to be effective in surveying a variety of carnivore species in an arid environment (Edwards, Gange \& Wiesel, 2016). It is recommended that camera traps are placed in random and passive sites for occupancy surveys (Rovero et al., 2013). However, due to the predicted low density of leopard and the arid environment, it is likely that a random placement of camera traps may result in mostly no leopard detections and thus placement of camera traps at predictable locations of activity may be necessary.

The final, model-averaged occupancy estimate had a relatively large SE; $\psi=0.64( \pm \mathrm{SE}$ 0.36), demonstrating a lack of precision for this estimate. Such a large standard error is indicative of unmodelled heterogeneity in the parameter (Mackenzie et al., 2006). As variance in repeat occurrence of leopard due to the proximity of other water sources is partialled out in the detection parameter, as observation error, it may leave unmodelled heterogeneity in occupancy, which is shown as a large standard error (Nielson et al., 2018; 
Stewart et al., in press). The concept requires further research to expand our understanding of its influence on occupancy models, but at this stage must be considered as a possibility. Following Mackenzie and Royle (2005), using the final, model-averaged occupancy and detection probabilities, the optimum number of sampling occasions suggested for occupancy surveys in such habitats is 10, two less than used in this study. However, a total of 112 camera trap sites, a seven-fold increase, would be needed to increase the precision of the occupancy estimate to having a SE of 0.05 . Such a high number of camera trap sites is unfeasible using the camera trap survey design of this study, given that only 18 artificial, permanent water sources are available within the park. One method of increasing the number of camera trap sites would be to place camera traps along minor roads and game trails, because no significant difference in leopard detection probability was found between artificial, permanent water sources and such paths in a similar, arid environment (Edwards, Gange \& Wiesel, 2016). However, keeping camera trap sites far enough apart to ensure spatial independence may mean surveying sites outside the park. Such an approach has the advantages of including both protected and non-protected areas, which could be incorporated into models as covariates to assess their influence on occupancy. Alternatively, spatiallyexplicit occupancy models may be used, e.g. Johnson et al. (2013), which are explicitly able to deal with spatial-autocorrelation occurring when units are not spatially independent.

Previous studies have suggested roads as having a variable influence on large felids. Whilst Rytwinski and Fahrig (2013) found most effects of roads to have a negative influence on population abundance, numerous other studies have reported a preferred use of roads by large felids (e.g. Harmsen et al., 2010). Several studies have also documented intersexual differences in responses to roads by carnivores, with females avoiding roads, and males either utilising them or remaining unaffected by their presence (Mace et al., 1996; Maehr, 1997; Conde et al., 2010). Negative effects of roads on carnivores may include increased mortality, population fragmentation and disturbance by traffic (Rytwinski \& Fahrig, 2013), as well as reductions in female reproductive success (Kerley et al., 2002). In this study, distance to access road was the covariate having the greatest influence on occupancy, with a positive association seen between site occupancy and distance to access road. In contrast, Mann, O'Riain \& Parker (2014) found leopard to show a strong preference for roads in the Little Karoo, South Africa. However, these roads were seldom used in comparison to those in GCP, which provide the only access routes to the three tourist lodges in the park. Such a difference may thereby suggest that the volume of traffic on roads may be the key factor in 
the influence they have on leopard. If so, the addition of new access roads may have a detrimental effect on the leopard population of the park.

Site elevation was shown to have the second strongest influence on occupancy, with a negative beta coefficient value, indicating sites at lower altitudes are more likely to be occupied by leopard. Such a result was not expected given the recorded preference for higher elevations by leopard (Nowell \& Jackson, 1996). This preference has been linked to an increased likelihood of hunting success, and the avoidance of dominant competitors such as lions Panthera leo (Pitman, Swanepoel \& Ramsay, 2012). However, Abade, Macdonald \& Dickman (2014) found leopards to show a preference for lower altitudes using a habitat suitability analysis in the Ruaha National Park in Tanzania. Abade, Macdonald \& Dickman (2014) suggested such a preference may be due to a higher distribution of the preferred prey species of leopard at lower altitudes, concentrated around perennial water sources. The same may be true for prey within the GCP. Alternatively, the absence of dominant leopard competitors may allow leopards to use mainly lower elevations. This result suggest further research focusing on the elevation preferences of leopards in arid environments, where dominant competitors are absent.

No individual leopard was detected visiting more than one site throughout the entire dataset. However, this may have been masked by the high proportion $(0.42)$ of blurry images unsuitable for individual identification, in combination with the use of only one camera trap per site which frequently produced a clear photo of only one flank of an individual leopard. Such factors also prevented the use of Stanley and Burnham's (1999) closure test to check for demographic closure occurring within the study period. When placing camera traps at artificial water sources and roads, Edwards et al. (2015) recorded $8.6 \mathrm{~km}$ and $13.76 \mathrm{~km}$ moved by individuals between cameras on commercial farmlands approximately $200 \mathrm{~km}$ northwest of the GCP. Such results suggest leopards within GCP may be visiting multiple water points, violating the assumption of spatial independence of sites. In such cases the occupancy results may be interpreted as a measure of habitat use, rather than 'true occupancy' (Mackenzie, 2005). The result of detection probability being positively associated with distance from the nearest neighbouring water source, may have also been biased by individuals using adjacent water points. As detection probability can be viewed as the degree of movement in and out of a site, that this was the only variable affecting detection probability is not surprising. Whilst excluding data from some sites to increase the nearest neighbour distance, thereby increasing the potential for spatial independence of sites, may be 
an option for studies with a large number of sites, it was not an option here given the relatively low number of sites $(n=15)$.

This study has demonstrated the ability of camera traps to produce information-rich datasets, which can be used to answer ecological questions ranging far beyond the focus of the study for which they were originally deployed, whilst also providing useful information regarding leopard which can be used to make informed management decisions on a national level. That leopard and Hartmann's mountain zebra belong to different guilds, with very different ecology, further highlights the wide range of data potentially produced by a single camera trap survey. Such potential, however, can only be utilised if all data from surveys are securely and properly stored and managed. This can require a relatively high investment of time to fully archive data, as well as large amounts of e-storage space (Witold et al., 2016). The benefits of such investments of time and resources have been demonstrated here, and give rise to the potential for increasing the 'return on investment' for many camera trap surveys. Therefore, investigators are urged to invest in the necessary resources for storing such non-target datasets, and to seek expertise able to perform relevant analyses.

\section{Acknowledgements}

We would like to thank the Gondwana Collection Namibia for making the camera trap data available to us for this analysis. We would also like to thank the Rufford Foundation, United Kingdom, the Whitley Fund for Nature, United Kingdom, and Parc Zoologique de Montpellier, France, for funding the Mountain Zebra Project. The Ministry of Environment and Tourism in Namibia is thanked for their assistance and providing a research permit to Morris Gosling (\#2100/2015). We also thank the Gondwana Canyon Park management and in particular the Gondwana Canyon Park staff for their participation in field data collection. Sarah Edwards received a post-doctoral bursary from the University of Pretoria, South Africa. We also thank Morris Gosling, Chris Brown, Jason Fisher for their comments on the manuscript.

\section{References}

Abade, L., Macdonald, D. W. \& Dickman, A. J. (2014). Using landscape and bioclimatic features to predict the distribution of lions, leopards and spotted hyaenas in Tanzania's Ruaha landscape. PLoS ONE, 9, e96261. 
Anderson, D. (2008). Model based inference in the life sciences: a primer on evidence. Springer, New York.

Anon (2015). Management and development plan for the Gondwana Canyon Park. Gondwana Canyon Park, Namibia.

Athreya, V., Odden, M., Linnell, J.D.C., Krishnaswamy, J. \& Karanth, K.U. (2013). Big cats in our backyards: persistence of large carnivores in a human-dominated landscape in India. PLoS ONE, 8, e57872.

Baker, J. (2004). Evaluation of closed capture-recapture methods to estimate abundance of Hawaiian monk seals. Ecological Applications, 14, 987-998.

Burnham, K.P. \& Anderson, D.R. (2002). Model selection and multimodel inference: a practical information-theoretic approach. Springer, New York.

Burton, A. C., Neilson, E., Moreira, D., Ladle, A., Steenweg, R., Fisher, J. T., Bayne, S. \& Boutin, S. (2015). Wildlife camera trapping: A review and recommendations for linking surveys to ecological processes. Journal of Applied Ecology, 52, 675-685.

Conde, D. A., Colchero, F., Zarza, H., Christensen, N. L., Sexton, J. O., Manterola, C., Chavez, C., Rivera, A., Azuara, D. \& Ceballos, G. (2010). Sex matters: modelling male and female habitat differences for jaguar conservation. Biological Conservation, 143, 19801988.

Cooch, E. \& White, G. (2006). Program MARK - a gentle introduction. Colorado State University, USA.

Dail, D. \& Madsen, L. (2011). Models for estimating abundance from repeated counts of an open metapopulation. Biometrics, 67, 577-587.

Edwards, S., Aschenborn, O., Gange, A. C. \& Wiesel, I. (2015). Leopard density estimates from semi-desert commercial farmlands, south-west Namibia. African Journal of Ecology, 54, 103-106. 
Edwards, S., Gange, A. C. \& Wiesel, I. (2016). An oasis in the desert: the potential of water sources as camera trap sites in arid environments. Journal of Arid Environments, 124, 304309.

Efford, M, G. \& Dawson, D.K. (2012). Occupancy in continuous habitat. Ecosphere, 3, 1-15.

Harmsen, B. J., Foster, R. J., Silver, S., Ostro, L. \& Doncaster, C. P. (2010). Differential use of trails by forest mammals and the implications for camera-trap studies: a case study from Belize. Biotropica, 42, 126-133.

Google (2016). Google Earth. http://earth.google.com/. Accessed September 2016.

Gosling, M. (2015). Mountain Zebra Project - 6th Progress Report. University of Newcastle, UK.

Guillera-Arroita, G., Ridout, M. S. \& Morgan, B. J. T. (2010). Design of occupancy studies with imperfect detection. Methods in Ecology and Evolution, 1, 131-139.

Harihar, A., Ghosh, M., Fernandes, M., Pandav, B. \& Goyal, S. P. (2010). Use of photographic capture-recapture sampling to estimate density of striped hyena (Hyaena hyaena): implications for conservation. Mammalia, 74, 83-87.

Hines, J.E. (2006). PRESENCE - Software to estimate occupancy and related parameters. USGS-PWRC. http://www.mbr-pwrc.usgs.gov/software/presence.html. Accessed December 2014.

Jacobson, A. P., Gerngross, P., Lemeris, J. R. Jr., Schoonover, R. F., Anco, C., BreitenmoserWursten, C., Durant, S.M., Farhadinia, M.S., Henschel, P., Kamler, J.F., Laguardia, A., Rostro-Gracia, S., Stein, A.B.\& Dollar, L. (2016). Leopard (Panthera pardus) status, distribution, and the research efforts across its range. PeerJ, 4, e1974.

Johnson, D. S., Conn, P. B., Hooten, M. B., Ray, J. C. \& Pond, B. (2013). Spatial occupancy models for large data sets. Ecology, 94, 801-808.

Jordan, M.J., Barrett, R.H. \& Purcell, K.L. (2011). Camera trapping estimates of density and survival of fishers Martes pennanti. Wildlife Biology, 17, 266-276. 
Karanth, K.U. \& Nichols, J.D. (1998). Estimation of tiger densities in India using photographic captures and recaptures. Ecology, 79, 2852-2862.

Kelly, J. \& Holub, E. (2008). Camera trapping of carnivores: trap success among camera types and across species, and habitat selection by species, on Salt Pond Mountain, Giles County, Virginia. Northeastern Naturalist, 15, 249-262.

Kerley, L.L., Goodrich, M.J., Miquelle, D.G., Smirnov, E.N., Quigley, H.B. \& Hornocker, M.G. (2002). Effects of roads and human disturbance on Amur tigers. Conservation Biology, $16,97-108$.

Krishnappa, Y. S. \& Turner, W. C. (2014). Software for minimalistic data management in large camera trap studies. Ecological Informatics, 24, 11-16.

Lindsey, P. A., Havemann, C. P., Lines, R., Palazy, L., Price, A. E., Retief, T. A., Rhebergen, T. \& van der Waal, C. (2013). Determinants of persistence and tolerance of carnivores on Namibian ranches: Implications for conservation on Southern African private lands. PloS One, 8 , e52458.

Linkie, M.Y., Dinata, A., Nugroho, A. \& Haidir, I.A. (2007). Estimating occupancy of a data deficient mammalian species living in tropical rainforests: sun bears in the Kerinci Seblat region, Sumatra. Biological Conservation. 20, 1599-1612.

Linkie, M., Guillera-arroita, G., Smith, J., Ario, A., Bertagnolio, G., Cheong, F., Clements, G.R., Dinata, Y., Duangchantrasiri, S., Fredriksson, G., Gumal, M.T., Horng, L.S., Kawanishi, K., Khakin, F.R., Kinnaird, M.F., Kiswayadi, D., Lubis, A.H., Lynman, A.J., Maryati, Maung, M., Ngoprasert, D., Novarino, W., O'Brien, T.G., Parakkasi, K., Peters, H., Priatna, D., Rayan, D.M., Seuaturien, N., Shwe, N.M., Steinmetz, R., Sugesti, A.M., Sunarto, S., Sunquist, M.E., Umponjan, M., Wibisono, H.T., Wong, C.C.T., Wong, C. C. T. \& Zulfahmi (2013). Cryptic mammals caught on camera: assessing the utility of range wide camera trap data for conserving the endangered Asian tapir. Biological Conservation, 162, $107-115$.

Long, R. A., Donovan, T. M., Mackay, P., Zielinski, W. J. \& Buzas, J. S. (2007). Comparing scat detection dogs, cameras, and hair snares for surveying carnivores. Journal of Wildlife Management, 71, 2018-2025. 
Long, R. A., Donovan, T. M., MacKay, P., Zielinski, W. \& Buzas, J. S. (2011). Predicting carnivore occurrence with non-invasive surveys and occupancy modelling. Landscape Ecology, 26, 327-340.

Mace, R.D., Waller, J.S., Manley, T.L., Lyon, I.J. \& Zuuring, H. (1996). Relationships amongst grizzly bears, roads and habitat in the Swan Mountains Montana. Journal of Applied Ecology, 33, 1395-1404.

Mackenzie, D.I., Nichols, J.D., Lachman, G.B., Droege, S., Royle, J.A. \& Langtimm, C.A. (2002). Estimating site occupancy rates when detection probabilities are less than one. Ecology, 83, 2248-2255.

Mackenzie, D.I. \& Royle, J.A. (2005). Designing occupancy studies: general advice and allocating survey effort. Journal of Applied Ecology, 42, 1105-1114.

Mackenzie, D.I., Royle, J.A., Pollock, K.H., Bailey, L.L. \& Hines, J.E. (2006). Occupancy estimation and modelling: inferring patterns and dynamics of species occurrence. Academic, London.

Maehr, D.S. (1997). Florida panther life and death of a vanishing carnivore. Island Press, Washington, D.C.

Mann, G. K. H., O’Riain, M. J. \& Parker, D. M. (2014). The road less travelled: assessing variation in mammal detection probabilities with camera traps in a semi-arid biodiversity hotspot. Biodiversity and Conservation, 24, 531-545.

Martin, R.B. \& du Meulenaer, T. (1988). Survey of the status of the leopard (Panthera pardus) in sub-Saharan Africa. CITES, Switzerland.

Mladenoff, D. J. \& Sickley, T. A. (1998). Assessing potential gray wolf restoration in the northeastern United States: a spatial prediction of favorable habitat and potential population levels. Journal of Wildlife Management, 61, 1-10.

National Drought Task Force (1997). National drought policy and strategy. National Drought Task Force, Windhoek, Namibia. 
Neilson, E.W., Avgar, T., Burton, A.C., Broadley, K. \& Boutin, S. (2018). Animal movement affects the interpretation of occupancy models from camera-trap surveys of unmarked animals. Ecosphere, 9, e02092. 101002/ecs2.2092.

Novellie, P. (2008). Equus zebra. The IUCN Red List of Threatened Species 2008:

Downloaded 6th March 2017.

O’Brien, T. G., Kinnaird, M. F. \& Wibisono, H. T. (2003). Crouching tigers, hidden prey: Sumatran tiger and prey populations in a tropical forest landscape. Animal Conservation, 6 , $131-139$.

O'Connell, A.F., Nichols, J.D. \& Karanth, K.U. (Eds.) (2011). Camera traps in animal ecology: methods and analyses. Springer, Japan.

Otis, D.L., Burnham, K.P., White, G.C. \& Anderson, D.R. (1978). Statistical inference from capture data on closed animal's populations. Wildlife Monographs, 62, 3-135.

Pamplin, F.A. (2013). Optimising the value of by-catch from Lynx lynx camera trap surveys in the Swiss Jura region. MSc thesis, University of East Anglia, United Kingdom.

Pitman, R. T., Swanepoel, L. H. \& Ramsay, P. M. (2012). Predictive modelling of leopard predation using contextual global positioning system cluster analysis. Journal of Zoology, $288,222-230$.

Quantum GIS Development Team (2015). QGIS. http://qgis/osgeo/org.

R Development Core Team (2014). R: A Language and Environment for Statistical Computing. Version 3.1.1. R Foundation for Statistical Computing, Vienna, Austria.

Rovero, F., Zimmermann, F., Berzi, D. \& Meek, P. (2013). "Which camera trap type and how many do I need?" A review of camera features and study designs for a range of wildlife research applications. Hystrix, 24, 148-156.

Rytwinski, T. \& Fahrig, L. (2013). Why are some animal populations unaffected or positively affected by roads? Oecologia, 173, 1143-1156. 
Srivathsa, A., Karanth, K. K., Jathanna, D., Kumar, N. S. \& Karanth, K. U. (2014). On a dhole trail: examining ecological and anthropogenic correlates of dhole habitat occupancy in the Western Ghats of India. PLoS ONE, 9, e98803.

Stanley, T.R. \& Burnham, K. P (1999). A closure test for time-specific capture-recapture data. Environmental and Ecological Statistics, 6, 197-209.

Stein, A. B., Aschenborn, O., Kastern, M., Andreas, A. \& Thompson, S. (2012). Namibia large carnivore atlas. Ministry of Environment and Tourism, Windhoek, Namibia.

Stein, A. B., Fuller, T. K., Destefano, S. \& Marker, L. L. (2011). Leopard population and home range estimates in north-central Namibia. African Journal of Ecology, 49, 383-387.

Stein, A. B. \& Hayssen, V. (2013). Panthera pardus (Carnivora: Felidae). Mammalian Species, 47, 30-48

Stratford, K. J., Weise, F., Melzheimer, J. \& De Woronin-Britz, N. (2016). Observations of servals in the highlands of central Namibia. Cat News, 64, 14-17.

Stewart, F., Fisher, J., Burton, A. \& Volpe, J. (2018). Species occurrence data reflect the magnitude of animal movements better than the proximity of animal space use. Ecosphere (in press).

Sunarto, S., Sollmann, R., Mohamed, A. \& Kelly, M. J. (2013). Camera trapping for the study and conservation of tropical carnivores. Raffles Bulletin Zoology, 28, 21-42.

Thirgood, S. \& Rabinowitz, A. (2005). The impact of human-wildlife conflict on natural systems. In Woodroffe, R., Thirgood, S. \& Rabinowitz, A. (Eds). People and wildlife: Conflict or coexistence? Cambridge University Press, Cambridge, UK.

Witold, J., Polish, B., Churski, M., \& Kuijper, D. P. (2016). TRAPPER: an open source webbased application to manage camera trapping projects manage camera trapping projects . Methods in Ecology and Evolution, 7, 1209-1216. 